I'Éléphant d'Afrique; elle est connue de la Haute Côte d'Ivoire, de la Côte d'Or, du lac Tchad, du Congo Belge et de l'Uganda.

Gedoelst (Revue Zoologique Africaine, IV, fasc. 2, [décembre 1916], p. 15̋8) a donné le nom de Cobboldia parumspinosa à des larves décrites par R. Blanchard (Ann. Soc: entom. France, LXII [1893], Bull., pp. 130-132, fig. 4) et recueillies par KinK en Zambésie. Dans notre Monographie de 1919, nous considérions avec doute ces parasites comme appartenant à $C$. loxodontis; en attendant un examen nouveau dés larves de KIRk, la question doit rester en suspens.

Les renseignements que nous possédons à l'heure actuelle sur les Diptères parasites de l'Éléphant d'Asie sont si pauvres qu'il serait du plus haut intérêt de faire une étude complète de ces insectes dans l'Inde, oủ leurs hòtes sont si communément tenus en captivité. Il est étonnant et bien regrettable qu'aucun des nombreux naturaistes qui ont séjourné dans ce pays, n'ait songé à faire des recherches à leur sujet.

\title{
Vichyia acyglossa, espèce et genre nouveaux de la famille des Milichiinae [Dipt. Muscidae]
}

par le $\mathrm{D}^{\mathrm{r}} \mathrm{J}$. Villeneuve.

J'ai pris à Vichy deux femelles d'une petite espèce (I millimètre ou guère plus) qui se place dans la 2 e section des Milichiinae selon HeNdel et appartient à un genre nouveau très voisin du genre Desmometopa Loew.

vichyia, nov. gen. -- Ce genre inédit se distingue de Desmometopa par : $\left.1^{\circ}\right)$ le profil de la tête semicirculaire, à péristome assez étroit, sans vibrisses audessus de la grande; $2^{\circ}$ ) les antennes insérées au-dessus du milieu des yeux ct ayant le $3^{\mathrm{e}}$ article volumineux et presque orbiculaire; $3^{\circ}$ ) la trompe très longue, géniculée et terminée en pointe, sans labelles $; 4^{\circ}$ ) la côte de l'aile ne présentant pas à sa base des cils raides, mais fins comme sur le reste de son étendue; enfin, les nervures transverses sont

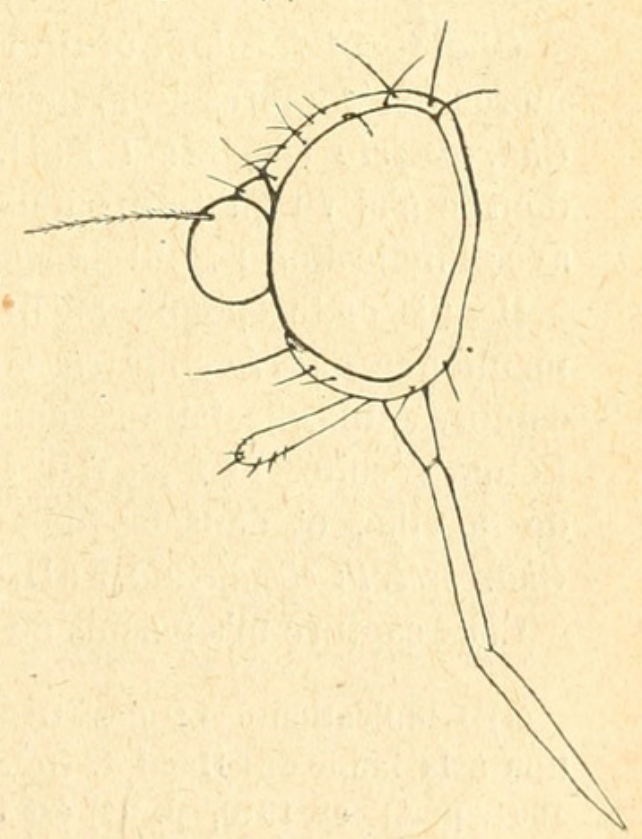

Fig. 1. - Vichyia acyglossa, n. sp. Tête vue de profil. 
beaucoup plus rapprochées, leur écart n'étant que la moitié de leur distance chez Desmometopa.

Chez Vichyia, les soies postverticales croisées sont aussi développées que les soies du vertex. Les soies du front ont d'ailleurs le même arrangement que chez Desmometopa; il y a, de même, 2 soies orbitaires postérieures tournées en dehors; mais les deux rangées internes des petites soies croisées ne sont pas portées par des bandelettes chitinisées, distinctes du reste du front par leur relief ou leur coloration. Le chète antennaire est épaissi à sa base, très brièvement cilié ailleurs. Les mésopleures sont nus. Soies du thorax et du scutellum comme chez Desmometopa.

V. acyglossa, n. sp. - o . D'un noir un peu brillant; abdomen mat avec la pilosité couchée. Front et antennes d'un noir de suie; clypéus obscur; palpes et trompe noirâtres; yeux nus. Pattes brunes; ailes à peine grises, balanciers épais et brunis.

Vole en mai-juin.

\section{Note sur deux espèces de Ghartoscirta Stâl}

[Hem. Acanthimde]

par le $D^{r}$ Maurice Royer.

Ghartoscirta elegantula Fall. - Dans son " Synopsis des Hémiptères Hétéroptères de France, $3^{e}$ partie (1880), p. 20 ə̋ ^, Putos indique Chartoscirta elegantula Fall. comme " espèce de l'Europe boréale, dont je n'ai vu qu'un exemplaire de France dans la collection Signoret avec l'indication : Mont-de-Marsan ".

Depuis cette époque, aucun des Catalogues régionaux français ne mentionne Chartoscirta elegantula F all. ( $\left.{ }^{(}\right)$et je n'en connais d'autre capture que celle faite récemment dans des détritus d'inondations du Loing, récoltés le $\boldsymbol{z}$ janvier 1920 à Moret (Seine-et-Marne), en amont de la ville, et dans lesquels se trouvait un spécimen du rarissime Chartoscirta elegantula $\mathrm{F}$ all.

Cette capture m'a permis de constater que si, comme l'indique Puton,

(1) L'indication " Landes ( $D^{\mathrm{r}}$ Gober $\mathrm{r}$ ) 》 donnée par Lambertie (Contribution à la faune des Hémipt. Hét. Cic. et Psyllides du sud-ouest de la France, 1901, p. 41 et 1910 , p. 44) est fausse. Les cinq individus de la collection Gobert qui sont piqués au-dessous de l'étiquette elegantula $\mathrm{F}$ all. sont des c. cincta H.-S.! 


\section{$2 \mathrm{BHL}$ Biodiversity Heritage Library}

Villeneuve, Joseph. 1920. "Vichyia acyglossa, espèce et genre nouveaux de la famille des Milichiinae [Dipt. Muscidae]." Bulletin de la Société entomologique de France 1920, 69-70.

View This Item Online: https://www.biodiversitylibrary.org/item/34136

Permalink: https://www.biodiversitylibrary.org/partpdf/93017

\section{Holding Institution}

Smithsonian Libraries

\section{Sponsored by}

Smithsonian

\section{Copyright \& Reuse}

Copyright Status: NOT_IN_COPYRIGHT

This document was created from content at the Biodiversity Heritage Library, the world's largest open access digital library for biodiversity literature and archives. Visit BHL at https://www.biodiversitylibrary.org. 